\title{
A simple empirical redshift indicator for gamma-ray bursts
}

\author{
J.-L. Atteia ${ }^{\star}$ \\ Laboratoire d'Astrophysique, Observatoire Midi-Pyrénées, 31400 Toulouse, France \\ Received 3 April 2003 / Accepted 23 June 203
}

\begin{abstract}
We propose a new empirical redshift indicator for gamma-ray bursts. This indicator is easily computed from the gamma-ray burst spectral parameters and its duration, and it provides "pseudo-redshifts" accurate to a factor two. Possible applications of this redshift indicator are briefly discussed.
\end{abstract}

Key words. gamma-rays: bursts

\section{Introduction}

Gamma-ray bursts (GRBs) are huge stellar explosions which have been observed at redshifts ranging from 0.0085 to 4.5 . While GRBs are in principle detectable out to very large redshifts $(z=10-20$, Lamb \& Reichart 2000), redshifts measured to date do not exceed 4.5. The method most frequently used to measure GRB redshifts is to find a visible afterglow, and to identify absorption lines in its spectrum, caused by the gas in the GRB host galaxy. The redshift of the host can also be measured at late times from the host emission lines, when the afterglow has faded below detection. Another, less frequent, method uses X-ray lines detected in the X-ray afterglows of some GRBs. The absence of GRB detection beyond $z=5$ could be explained by the fact that the afterglows of such distant GRBs must be searched for in the infrared, due to the Lyman alpha cutoff. The difficulty to measure spectroscopic redshifts led various authors to propose alternate ways to determine GRB redshifts. Norris et al. (2000) and Reichart et al. (2001) have found empirical luminosity estimators based on GRB light curves. Such luminosity estimators can be used to infer the intrinsic luminosity of individual GRBs, and consequently their redshifts. While these estimators cannot be used to obtain precise redshifts for individual GRBs, they are useful to derive statistical properties of the GRB population.

Redshift estimators based on the gamma-ray data only present two distinctive advantages: they provide redshift estimates for most GRBs detected in gamma-rays, and they do not require extensive follow-up campaigns involving large telescopes on the ground or in space. Important issues can be addressed with moderatly accurate redshifts, like the amount of energy released by GRBs in gamma-rays, the luminosity function of GRBs, or the history of stellar formation at high redshifts.

\footnotetext{
* e-mail: atteia@ast.obs-mip.fr
}

We propose here a new method to obtain redshift indicators for GRBs from gamma-ray observations. Our method is calibrated with 17 GRBs detected with BeppoSAX (Boella et al. 1997) and HETE (Ricker et al. 2001). In the following we call the redshifts inferred from our redshift indicator "pseudo-redshifts". Pseudo-redshifts have the advantage of being very easily computed. In addition to the possible applications already mentioned, pseudo-redshifts may become a useful tool to quickly identify high-redshift GRBs.

\section{An empirical redshift indicator}

Finding redshift indicators for GRBs based on the gamma-ray data alone has always faced the problem of the large intrinsic dispersion of GRB properties. This intrinsic dispersion prevents the determination of the redshifts of individual GRBs. With the measure of an increasing number of GRB redshifts it appeared, however, that several properties of GRBs are correlated with the isotropic-equivalent energy radiated in gammarays (called $E_{\text {rad }}$ in the following). For instance, the correlation of the spectral hardness with $E_{\text {rad }}$ has been suspected for a long time (see e.g. Atteia 2000; Lloyd et al. 2000). It has only been demonstrated recently by Amati et al. (2002) for 12 GRBs with known redshifts. The correlation of the duration with $E_{\text {rad }}$ is discussed in Lee et al. (2000). These correlations have led some authors to propose using the observed GRB properties to infer $E_{\text {rad }}$, and then to deduce the redshift from the comparison of the observed fluence with $E_{\text {rad }}$. Norris et al. (2000), for instance, estimate $E_{\text {rad }}$ from the magnitude of the time lags between a high energy band and a low energy band. Reichart et al. (2001) estimate $E_{\text {rad }}$ from the variability of the light curve. We propose and test here another approach: we search a quantity which depends little on $E_{\text {rad }}$, and which has a small intrinsic dispersion which does not blur the dependence on redshift. Starting from empirical considerations, We find such a quantity essentially based on the spectral characteristics of GRBs. 


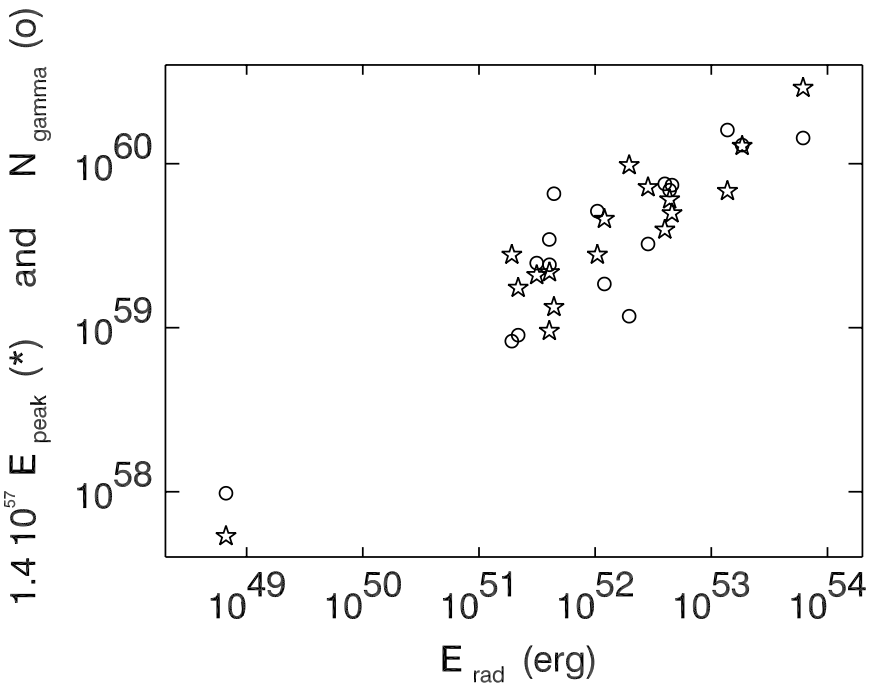

Fig. 1. Dependence of two characteristic GRB quantities on $E_{\mathrm{rad}}$, the isotropic-equivalent energy radiated in gamma-rays. The stars show the dependence of the intrinsic peak energy (multiplied here by $1.4 \times 10^{57}$ ). The circles show the dependence of the isotropicequivalent number of photons emitted by the source.

GRB energy spectra are well fit with the so-called GRB model, consisting of two smoothly connected power laws (Band et al. 1993). In the following, $\alpha$ is the index of the lowenergy power law, $\beta$ the index of the high-energy power law, and $E_{0}$ is the break energy. With this parametrization, the peak energy of the $v f_{v}$ spectrum is $E_{\mathrm{p}}=E_{0} \times(2+\alpha)$. $E_{\mathrm{p}}$ is well defined for $\alpha \geq-2$ and $\beta<-2$.

Our method is based on the recent finding by Amati et al. (2002) of a correlation between the intrinsic (redshift corrected) $E_{\mathrm{p}}$ of 12 GRBs with known redshifts, and $E_{\text {rad }}$, their isotropic-equivalent energy radiated in gamma-rays. According to Amati et al., $E_{\mathrm{p}}$ is roughly proportional to the square-root of $E_{\text {rad }}$. Since $\alpha$ and $\beta$ do not vary too much from burst to burst, and since the energy radiated in gamma-rays is more or less the product of the number of photons by their typical energy, we make the assumption that the isotropic-equivalent number of photons in a GRB, $N_{\gamma}$, is also roughly proportional to the square-root of $E_{\text {rad }}$. For this study, we define $N_{\gamma}$ as the number of photons below the break, integrated from $E_{\mathrm{p}} / 100$ to $E_{\mathrm{p}} / 2$.

Figure 1 shows $E_{\mathrm{p}}$, the intrinsic peak energy, and $N_{\gamma}$, the isotropic-equivalent number of photons as a function of $E_{\mathrm{rad}}$ for a sample of 17 GRBs detected by BeppoSAX, BATSE, and HETE. The main characteristics of these GRBs are given in Table 1, along with references for their spectral parameters. The redshifts have been taken from J. Greiner's GRB page at http://www.mpe.mpg.de/ jcg/grbgen.html (except for GRB 020124, which comes from Hjorth et al. 2003). Figure 1 shows that, as we suspected, $E_{\mathrm{p}}$ and $N_{\gamma}$ have roughly the same dependence on $E_{\text {rad. }}$. We can thus go one step further with our main conjecture: we suppose that the ratio $N_{\gamma} / E_{\mathrm{p}}$ is almost independent of $E_{\text {rad }}$, and can be used as a redshift indicator. Figure 2 shows that indeed the ratio $N_{\gamma} / E_{\mathrm{p}}$ shows very little dependence on $E_{\text {rad }}$, confirming our conjecture. This is not sufficient, however, to make it a correct redshift indicator. The critical issue is to find an indicator which has a small

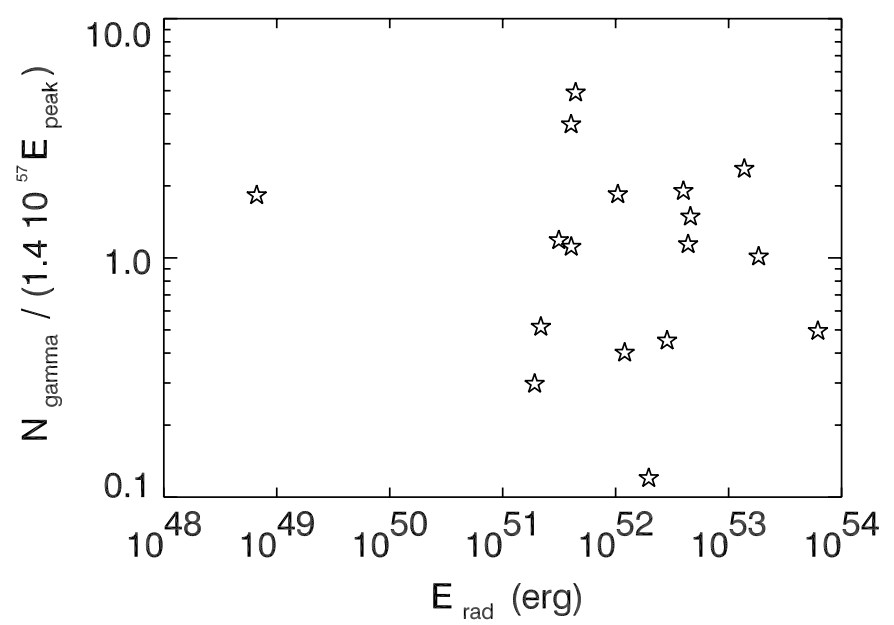

Fig. 2. The ratio $N_{\gamma} / E_{\mathrm{p}}$ (see text) as a function of $E_{\mathrm{rad}}$. This figure illustrates the weak dependence of $N_{\gamma} / E_{\mathrm{p}}$ with $E_{\mathrm{rad}}$.

Table 1. Observed properties of 17 GRBs with known redshift. The ten columns give the GRB name, the duration $T_{90}$ in seconds, the three spectral parameters $\left(\alpha, \beta\right.$, and $\left.E_{0}\right)$, the gamma-ray fluence $S_{\gamma}$ in units of $10^{-6} \mathrm{erg} \mathrm{cm}^{-2}$, the spectroscopic redshift $z$, the pseudo-redshift $\hat{z}$, the ratio $\hat{z} / z$, and a reference for the spectral parameters.

\begin{tabular}{llllllllllll}
\hline \hline Name & $T_{90}$ & $\alpha$ & $\beta^{a}$ & \multicolumn{3}{c}{$E_{0}$} & $S_{\gamma}$ & $z$ & $\hat{z}$ & $\hat{z} / z$ & ref. \\
& $\mathrm{seV}$
\end{tabular}

${ }^{a} \beta$ has been frozen to -2.3 for HETE GRBs 010921 to 030228 . 1 Amati et al. (2002). Fluence measured in the range 40-700 keV. 2 Andersen et al. (2000). Fluence measured in the range $28-1800 \mathrm{keV}$.

3 Barraud et al. (2003). Fluence measured in the range $30-400 \mathrm{keV}$. 4 Sakamoto et al. (2003). Fluence measured in the range 7-30 keV. 5 Crew et al. (2003). Fluence measured in the range $7-30 \mathrm{keV}$. $6 \mathrm{Lamb}$ et al. (2003). Fluence measured in the range $30-400 \mathrm{keV}$. 7 Vanderspek et al. (2003). Fluence measured in the range 7-30 keV.

dependence on $E_{\text {rad }}$, a strong dependence on the redshift, and a not too strong intrinsic dispersion. This issue is discussed in the next section. 


\subsection{Definition of the redshift indicator}

The theoretical considerations in the previous section are based on the study of intrinsic GRB properties. Defining a redshift indicator implies that we do not know the redshifts of the GRBs which are being studied, but only their observed properties. To keep in mind this difference, in all the following we use capital letters for intrinsic quantities, and lower case for observed quantities.

As discussed earlier, the best redshift indicator is not necessarily the one with the smallest intrinsic dispersion, but rather the one which has the best combination of a small intrinsic dispersion and a large dependence on redshift. Relying on the analysis of the previous section, we propose to base our redshift indicator on $n_{\gamma} / e_{\mathrm{p}}$ the ratio of the observed number of photons in the GRB on the observed peak energy. We tried various simple combinations of GRB parameters, all involving the ratio $n_{\gamma} / e_{\mathrm{p}}$, and found that $X=n_{\gamma} / e_{\mathrm{p}} / \sqrt{t_{90}}$ has the right combination of properties for a redshift indicator. In this equation $e_{\mathrm{p}}$ is the observed peak energy, $n_{\gamma}$ the observed number of photons between $e_{\mathrm{p}} / 100$ and $e_{\mathrm{p}} / 2$, and $t_{90}$ the observed duration. We do not claim here that $X$ is definitely the best redshift indicator, we nevertheless believe that it is sufficiently good to deserve further discussion.

We derive pseudo-redshifts from the measure of $X$ in the following way: in a first step we compute the theoretical evolution of $X$ with redshift; then we invert this relation to derive a pseudo-redshift from the observed value of $X$. The evolution of $X$ with redshift can be written as

$X=A \times f(z)$

$A$ is a constant of normalization, and $f$ describes the evolution of $X$ with redshift for a "standard" GRB $(\alpha=-1.0$, $\beta=-2.3$, and $\left.E_{0}=250 \mathrm{keV}\right)$ in a "standard" universe $\left(H_{0}=\right.$ $\left.65 \mathrm{~km} \mathrm{~s}^{-1} \mathrm{Mpc}^{-1}, \Omega_{0}=0.3, \Omega_{\Lambda}=0.7\right)$. GRB spectral parameters are not critical here, because we have shown in the previous section that the ratio $N_{\gamma} / E_{\mathrm{p}}$ does not vary much from burst to burst. The normalization constant $A$ has been chosen to have about the same number of GRBs below and above the theoretical curve in Fig. $3(A=60)$.

Pseudo-redshifts $\hat{z}$ are then defined by

$\hat{z}=\frac{1}{A} \times f^{-1}(X)$.

Their use as redshift indicators is discussed below.

\subsection{Evaluation of pseudo-redshifts}

Figure 3 shows the values of $X$ as a function of $z$, for the 17 GRBs of Table 1. This figure displays a clear anticorrelation between the two quantities. The dotted line indicates the theoretical dependence $X=A \times f(z)$. The coefficient of correlation between $z$ and $X$ is -0.875 , corresponding to a correlation significant at the level of 4.9 sigmas using Fisher's Z transformation. We consider that this anticorrelation provides a good support to our intention of using $X$ as a redshift indicator, and we use the Eq. (1) above to compute the pseudo-redshifts of GRBs in Table 1. Table 1 gives the values

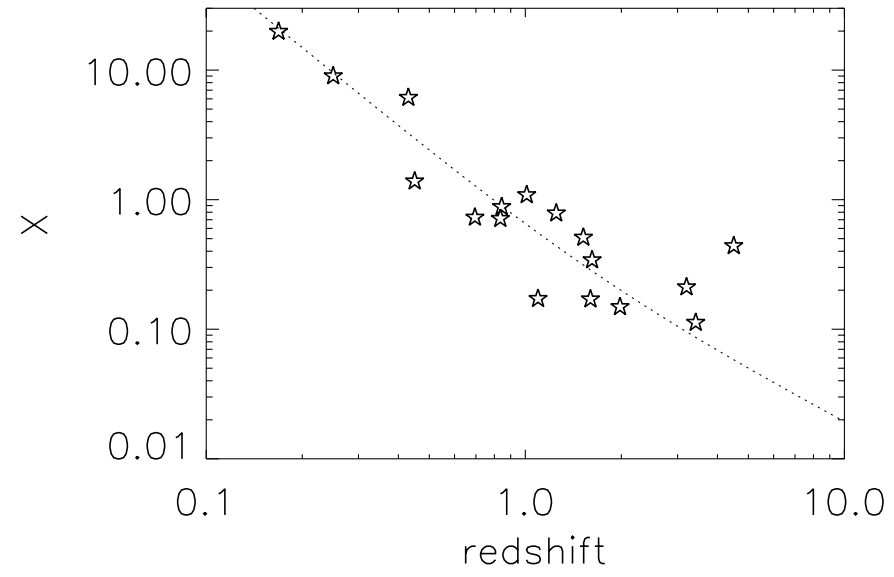

Fig. 3. Correlation of $X=n_{\gamma} / e_{\mathrm{p}} / \sqrt{t_{90}}$ (see text ) with the measured redshifts of 17 GRBs. The isolated star at $z=4.5$ is GRB 000131. The dotted line shows the theoretical relation between $\hat{z}$ and $z$.

of $z, \hat{z}$, and their ratio, for the 17 GRBs with known redshift used in our analysis ${ }^{1}$. It shows that $\hat{z}$ is usually within a factor of two of $z$, except for GRB 000131 (at $z=4.5$ ), for which $z$ and $\hat{z}$ differ by a factor 3.3 . This discrepancy could be the consequence of the low quality of our redshift indicator for this burst (most probably) or of a problem with the measure of the spectral parameters of this GRB or of its redshift. It might also indicate that the relation between $z$ and $\hat{z}$ is only working (or calibrated) to $z=3.5$. Because this event is clearly an outlier, we recomputed the coefficient of correlation between $z$ and $X$ without it. We find a coefficient of correlation of -0.927 , corresponding to a correlation significant at the level of 6.1 sigmas using Fisher's Z transformation.

We conclude that the intrinsic dispersion of $X$ is not such that it prevents its use as a redshift indicator. We prefer the term redshift indicator than redshift estimator, because the ratio of $\hat{z}$ over $z$ varies too much for a true redshift estimator. In the following we use the name pseudo-redshifts for $\hat{z}$. Because $\hat{z}$ was derived from a purely empirical approach, we expect that an approach based on a physical treatment of GRB emission might provide a better redshift estimator. While pseudo-redshifts are a potentially useful tool, they deserve a word of caution here because we do not know how observational biases affect Fig. 3. We note for instance that GRBs with spectroscopic redshifts certainly represent a biased sample. In addition the relations linking the GRB properties (from which we compute $X$ ) to those of their afterglows (from which we measure $z$ ) are far from being understood. Anyone using this tool should thus keep in mind that Fig. 3 provides a biased view of the true distribution of GRBs in the $(z, X)$ plane.

\footnotetext{
1 Two GRBs in Amati et al. (2002) are not included in Table 1 because they do not have spectroscopic redshifts. The redshift of GRB 980326 was estimated to be 1 from the observation of a supernova bump in the late light curve of the afterglow (Bloom et al. 1999), and we find $\hat{z}=1.05$. The redshift of GRB 000214 was estimated to be 0.42 from the observation of an iron line in its X-ray afterglow (Antonelli et al. 2000), and we find $\hat{z}=0.39$.
} 
Table 2. Observed properties of 18 GRBs with no measured redshift. The eight columns give the name of the GRB, the time of arrival, the duration $T_{90}$ in seconds, the three spectral parameters $(\alpha, \beta$, and $E_{0}$ ), the gamma-ray fluence $S_{\gamma}$ in units of $10^{-6} \mathrm{erg} \mathrm{cm}^{-2}$ in the range $30-400 \mathrm{keV}$, the pseudo-redshift $\hat{z}$, and a comment on the eventual detection of an afterglow (XRR stands for X-Ray Rich GRB, OA, XA, and RA, respectively for Optical Afterglow, X-ray Afterglow, and Radio Afterglow).

\begin{tabular}{llllllll}
\hline \hline Name & $\begin{array}{l}\text { Time } \\
\text { SOD }\end{array}$ & $T_{90}$ & $\alpha$ & $\begin{array}{l}E_{0} \\
\mathrm{keV}\end{array}$ & $S_{\gamma}^{\mathrm{e}}$ & $\hat{z}$ & Comment \\
& \multicolumn{1}{c}{$\mathrm{s}^{2}$} & & \\
\hline 001225 & 25759 & 32.3 & -1.17 & 283 & 114 & 0.64 & \\
010126 & 33162 & 7.7 & -1.06 & 115 & 3.0 & 1.6 & \\
$010326 A$ & 11701 & 23.0 & -.894 & 260 & 16 & 2.8 & \\
010613 & 27235 & 152. & -1.40 & 176 & 20.3 & 0.85 & \\
010629 & 44468 & 15.1 & -1.17 & 59 & 2.6 & 0.76 & XRR \\
010928 & 60826 & 48.3 & -.623 & 260 & 21 & 4.9 & \\
020113 & 7452 & 1.31 & -0.46 & 239 & 1.3 & 2.3 & Short/Hard \\
020127 & 75444 & 9.3 & -1.19 & 156 & 0.9 & 3.9 & XA, RA, host \\
020214 & 67778 & 27.4 & -.256 & 176 & 93 & 1.7 & \\
020305 & 42925 & 250. & -.861 & 143 & 10.4 & 4.6 & OA, host \\
020331 & 59548 & 56.5 & -.922 & 120 & 4.5 & 3.4 & OA \\
020418 & 63789 & 7.54 & -1.10 & 240 & 13.9 & 1.3 & \\
020531 & 1578 & 1.15 & -1.10 & 810 & 1.2 & 13.5 & Short/Hard \\
020801 & 46721 & 336. & -1.32 & 116 & 16.3 & 0.95 & \\
020812 & 38503 & 27.5 & -1.03 & 125 & 2.3 & 3.4 & \\
020819 & 53855 & 33.6 & -1.03 & 94 & 5.4 & 1.5 & RA, host \\
021016 & 37740 & 81.6 & -.98 & 132 & 11.3 & 2.1 & \\
021104 & 25262 & 19.7 & -1.0 & 27 & 0.3 & 1.6 & XRR \\
\hline
\end{tabular}

\section{An example of using pseudo-redshifts}

In this section we compute the pseudo-redshifts of $18 \mathrm{GRBs}$ detected by HETE/FREGATE, whose spectral parameters are given in Barraud et al. (2003). We compare them with the pseudo-redshifts of $8 \mathrm{GRBs}$ with known redshifts in Table 1 in order to assess the role of the redshift in the non-detection of the afterglows for these GRBs. The pseudo-redshifts of these 18 GRBs are given in Table 2.

The first remark is that short/hard GRBs should probably not be integrated in our framework. GRB 020531 for instance has a low $X$ value, which results in an unrealistically high pseudo-redshift. Having no redshift for short/hard bursts we cannot evaluate, and eventually calibrate, our redshift indicator for these bursts. The two shord/hard GRBs of our sample, GRB 020113 and GRB 020531, are thus excluded from the rest of our analysis.

The median pseudo-redshift of long GRBs in Table 2 is 1.65 , while it is only 0.88 for the 8 FREGATE GRBs with a measured redshift in Table 1. If we believe the correlation between the pseudo-redshifts and the true redshifts, this indicates that the redshift certainly plays a role in the non-detection of the afterglows of FREGATE GRBs, even if this is not the only factor as emphasized by Crew et al. (2003).

While pseudo-redshifts can be useful for statistical analyses, the information they convey is probably not meaningful for individual GRBs. We believe however that pseudo-redshifts could become a useful tool to quickly identify high redshift
GRBs from the gamma-ray data alone. A first step in this direction is obviously to prove the validity of pseudo-redshifts for this task. GRB 020127 may appear as a good test case in this context because it has a high pseudo-redshift $(\hat{z}=3.9$ in Table 2), a possible X-ray afterglow, a possible radio afterglow, and a candidate host galaxy (Fox et al. 2002a, 2002b, 2002c). If the host candidate is at a redshift of about 3 , this would strengthen the validity of pseudo-redshifts as a tool for the quick identification of high redshift GRBs.

\section{Conclusion}

We propose an empirical redshift indicator for GRBs, which is easily computed from the gamma-ray data alone and provides "pseudo-redshifts" accurate to a factor of two. Despite their moderate accuracy, we believe that their easy computation will make these pseudo-redshifts useful in future GRB studies. Their possible applications include a statistical comparison of the distance distribution of distinct GRB populations, constraints on the star formation rate at high redshifts, and the fast identification of remote GRBs, with redshifts beyond three.

The usefulness of these pseudo-redshifts will ultimately depend on the confirmation of their accuracy, which will be tested as a larger number of GRBs with known redshifts become available.

Acknowledgements. The author is grateful to R. Mochkovitch for insightful discussions on pseudo-redshifts and to C. Barraud for helpful suggestions. JLA acknowledges the use of J. Greiner GRB page at http: //www . mpe.mpg.de $/{ }^{j} \mathrm{jcg} / \mathrm{grbgen} . \mathrm{html}$, and useful comments from the referees.

\section{References}

Andersen, M. I., Hjorth, J., Pedersen, H., et al. 2000, A\&A, 364, L54 Amati, L., Frontera, F., Tavani, M., et al. 2002, A\&A, 390, 81 Antonelli, L. A., Piro, L., Vietri, M., et al. 2000, ApJ, 545, L39 Atteia, J-L. 2000, A\&A, 353, L18

Band, D., Matteson, J., Ford, L., et al. 1993, ApJ, 413, 281

Barraud, C., Olive, J-F., Lestrade, J. P., et al. 2003, A\&A, 400, 1021

Bloom, J., Kulkarni, S., Djorgovski, S., et al. 1999, Nature, 401, 453

Boella, G., Butler, R., Perola, G., et al. 1997, A\&AS, 122, 299

Crew, G. B., Lamb, D. Q., Ricker, G. R., et al. 2003, ApJ, submitted

Fox, D. W. 2002a, GCN 1249

Fox, D. W., \& Frail, D.A. 2002b, GCN 1250

Fox, D. W., Djorgovski, S.G., \& Kulkarni, S.R. 2002c, GCN 1306

Hjorth, J., et al. 2003, Proc. of the 3rd workshop Gamma-Ray Bursts in the Afterglow Era, Roma 2002

Lamb, D. Q., \& Reichart, D. E. 2000, ApJ, 536, 1

Lamb, D. Q., Sakamoto, T., Atteia, J.-L., et al. 2003, ApJ, submitted Lee, A., Bloom, E. D., \& Petrosian, V. 2000, ApJS, 131, 21

Lloyd, N. M., Petrosian, V., \& Mallozzi, R. S. 2000, ApJ, 534, 227

Norris, J., Marani, G., \& Bonnell, J. 2000, ApJ, 534, 248

Reichart, D., Lamb, D., Fenimore, E., et al. 2001, ApJ, 552, 57

Ricker, G., Atteia, J-L., Crew, G., et al. 2001, AIP Conf. Proc., 662, 3 Sakamoto, T., Lamb, D. Q., Suzuki, M., et al. 2003, ApJ, submitted Vanderspek, R., Lamb, D., Sakamoto, T., et al. 2003, ApJ, submitted 